\title{
Efficacy of Tamsulosin in Relieving Double-J Stent-related Symptoms: a Randomized Controlled Study
}

Thakur DK, Chapagain S, Luitel BR ,Chalise PR ,Sharma UK, Gyawali PR

Urology Division, Department of Surgery, Tribhuvan University Teaching Hospital, Kathmandu, Nepal

Correspondence :Dr Deepak K Thakur MCh(Urology)Resident, Urology Division, Department of Surgery, Tribhuvan University Teaching Hospital, Kathmandu, Nepal.

email:yoursdeepak2000@gmail.com

\begin{abstract}
Introduction: Ureteral stent placement is an increasingly common procedure in urological practice.They are used for both prevention and treatment of ureteral obstruction. Despite improved design and materials, many patients still develop stent-related symptoms which commonly affect quality of life and sometimes necessitate early removal. Tamsulosin improves stent-related symptoms and quality of life, and can be applied in routine clinical practice. In the present study, the effect of Tamsulosin in improving double-J stent-related symptoms and quality of life following ureteral stent placement was studied.
\end{abstract}

Methods: This RCT was carried out in the Department of Surgery, Tribhuvan University Teaching Hospital, Kathmandu, Nepal from February 2015 to January 2016. Forty six patients were included in the study and randomized intoTamsulosin( $\mathrm{T})$ group and Control(C) group each having 23 patients. In addition to standard postoperative care, Tamsulosin group received $0.4 \mathrm{mg}$ Tamsulosin daily and Paraceramol on demand and control group received only Paracetamol. Stent related symptoms and quality of life was assessed by IPSS at discharge (day2) and at the time of DJ stent removal ( 2 weeks). Pain was evaluated by VAS and analgesic requirement was documented. Data were analysed using SPSS 20, chi-square test and Student's t- test was used. A p-value of $<0.05$ was considered significant.

Results: Mean age in Tamsulosin group was $37.96 \pm 12.98$ and Control group $36.43 \pm 10.99(p=0.67)$.There was no significant difference in IPSS $(p=0.141)$, QoL index $(p=0.089)$ and VAS $(p=0.59)$ in the two groups at the time of discharge.At the time of DJ stent removal,IPSS $(p<0.001)$, QoL index $(p<0.001)$,VAS $(p=0.004)$ and analgesic needed $(p<0.001)$ was significantly lower in $T$ group than in $C$ group.

Conclusion:Tamsulosin lowers stent related symptoms, pain and improves quality of life in patients with indwelling DJ stent though the effect is not immediate.

Key words: DJ stent,IPSS, Tamsulosin, VAS 